Research Article

\title{
When the Cartesian product of directed cycles is hyper-Hamiltonian
}

\author{
Zbigniew R. Bogdanowicz* \\ CCDC Armaments Center, Picatinny, New Jersey 07806, USA
}

(Received: 3 September 2021. Received in revised form: 22 November 2021. Accepted: 6 December 2021. Published online: 13 December 2021.)

(C) 2021 the author. This is an open access article under the CC BY (International 4.0) license (www.creativecommons.org/licenses/by/4.0/).

\begin{abstract}
Sufficient conditions for the Cartesian product of directed cycles to be hyper-Hamiltonian are given, and it is proved that only the Cartesian product of at least three directed cycles can be hyper-Hamiltonian.
\end{abstract}

Keywords: hyper-Hamiltonian graph; hypo-Hamiltonian graph; Cartesian product of directed cycles; Hamiltonian digraph.

2020 Mathematics Subject Classification: 05C38, $05 \mathrm{C} 45$.

\section{Introduction}

A Hamiltonian (a non-Hamiltonian, respectively) graph $G$ is hyper-Hamiltonian (hypo-Hamiltonian, respectively) if every vertex-deleted subgraph $G \backslash\{v\}$ is Hamiltonian. A Cartesian product $G=C_{n_{1}} \square C_{n_{2}} \square \cdots \square C_{n_{k}}$ of $k$ directed cycles $C_{n_{1}}, C_{n_{2}}, \ldots, C_{n_{k}}$ is the graph such that the vertex set $V(G)$ equals the Cartesian product $V\left(C_{n_{1}}\right) \times V\left(C_{n_{2}}\right) \times \cdots \times V\left(C_{n_{k}}\right)$ and there is an arc in $G$ from vertex $u=\left(u_{1}, u_{2}, \ldots, u_{k}\right)$ to vertex $v=\left(v_{1}, v_{2}, \ldots, v_{k}\right)$ if and only if there exists $1 \leq r \leq k$ such that there is an $\operatorname{arc}\left(u_{r}, v_{r}\right)$ in $C_{n_{r}}$ and $u_{i}=v_{i}$ for all $i \neq r$. In this paper we study when the Cartesian product of directed cycles is hyper-Hamiltonian. Hence, throughout the rest of this paper $C_{n_{i}}$ denotes a directed simple cycle of length $n_{i} \geq 2$. This topic has long related history in the literature.

In 1948, Rankin [7] gave an implicit necessary and sufficient condition for the existence of a Hamilton cycle in $C_{n_{1}} \square C_{n_{2}}$ based on embedding it on a torus. Later, Trotter and Erdős proved independently the result, that turned out to be a special case of Rankin's result, for the Cartesian product of two directed cycles as follows:

Theorem 1.1. [9] The Cartesian product of two directed cycles $C_{n_{1}} \square C_{n_{2}}$ contains a Hamilton cycle if and only if there exist positive integers $t_{1}$ and $t_{2}$ such that $\operatorname{gcd}\left(n_{1}, n_{2}\right)=t_{1}+t_{2}$ and $\operatorname{gcd}\left(n_{1}, t_{1}\right)=\operatorname{gcd}\left(n_{2}, t_{2}\right)=1$.

Subsequently, Thomassen [8] gave a sufficient condition for $C_{n_{1}} \square C_{n_{2}}$ to be hypo-Hamiltonian,

Theorem 1.2. [8] The Cartesian product of two directed cycles $C_{n_{1}} \square C_{n_{2}}$ is hypo-Hamiltonian if there exists positive integer $t$ such that $n_{2}=t \cdot n_{1}-1$.

In 1983, Penn and Witte extended Thomassen's result to a necessary and sufficient condition as follows:

Theorem 1.3. [6] The Cartesian product of two directed cycles $C_{n_{1}} \square C_{n_{2}}$ is hypo-Hamiltonian if and only if there is a pair of relatively prime positive integers $x$ and $y$ with $x n_{1}+y n_{2}=n_{1} n_{2}-1$.

In addition, Curran and Witte extended the result of Trotter and Erdös to the Cartesian product of $k$ directed cycles for $k \geq 3$.

Theorem 1.4. [3] For all $r \geq 3$ and all $n_{1}, n_{2}, \ldots, n_{r}>1$, there is a Hamilton cycle in $C_{n_{1}} \square C_{n_{2}} \square \ldots \square C_{n_{r}}$.

Finally, more focus in recent years has been on when the special families of graphs are hyper-Hamiltonian $[1,2,4,5]$.

In Section 2, we leverage Theorem 1.3 and we prove that $C_{n_{1}}^{\square} \square C_{n_{2}}$ is not hyper-Hamiltonian. In Section 3, we leverage Theorems 1.1, 1.2, 1.3, and 1.4, and we give the sufficient conditions for $C_{n_{1}} \square C_{n_{2}} \square \cdots \square C_{n_{k}}$ to be hyper-Hamiltonian if $k \geq 3$.

\footnotetext{
*E-mail addresses: zbigniew.bogdanowicz.civ@mail.mil, zrb2@aol.com
} 


\section{Cartesian product of two directed cycles}

In the following theorem we utilize the theory of torus knots, as was done in [6,7]. In particular, Penn and Witte [6] also used the torus knots approach in the proof of Theorem 1.3.

Theorem 2.1. $C_{n_{1}} \square C_{n_{2}}$ is not hyper-Hamiltonian.

Proof. Suppose $G=C_{n_{1}} \square C_{n_{2}}$ is hyper-Hamiltonian. Let $C_{G}$ be a Hamilton cycle in $G$ and $C_{H}$ be a Hamilton cycle in $H=G \backslash\{v\}$. Let $a_{i}$ be an arc in $G$ induced by $C_{n_{i}}$, where $i \in\{1,2\}$. Let $(s, t) \in Z \times Z$ be the knot class of $C_{H}$ considered as a knot on the torus. Clearly, there must be $s n_{1}$ occurrences of $a_{1}$ and $t n_{2}$ occurrences of $a_{2}$ in $C_{H}$. Because the length of $C_{H}$ is $n_{1} n_{2}-1$ then $s n_{1}+t n_{2}=n_{1} n_{2}-1$ must be satisfied. Hence, this excludes the case where $n_{1}, n_{2}$ are even. Furthermore, since $(s, t)$ is the knot class of a circuit $C_{H}, \operatorname{gcd}(s, t)=1$ must be satisfied too. So, by Theorem $1.3 G$ is hypo-Hamiltonian and $C_{G}$ does not exist - a contradiction that proves this theorem.

In addition, we obtain the following lemma that will be useful in Section 3.

Lemma 2.1. Let $G=C_{n_{1}} \square C_{n_{2}}$. G\\{v\} has a Hamilton cycle if and only if there is a pair of relatively prime positive integers $s$ and $t$ with $s n_{1}+t n_{2}=n_{1} n_{2}-1$.

Proof. Based on Theorems 1.3 and 2.1, $G \backslash\{v\}$ has a Hamilton cycle if and only if it is hypo-Hamiltonian. Hence, this lemma follows directly from Theorem 1.3.

\section{Cartesian product of at least three directed cycles}

First, we characterize when the Cartesian product of three directed cycles is hyper-Hamiltonian.

Theorem 3.1. $C_{n_{1}} \square C_{n_{2}} \square C_{n_{3}}$ is hyper-Hamiltonian if there is a permutation $\left(n_{1}^{\prime}, n_{2}^{\prime}, n_{3}^{\prime}\right)$ of $\left(n_{1}, n_{2}, n_{3}\right)$ and there exist positive integers $t_{1}, t_{2}, t_{3}, t_{4}$ so that $t_{1}+t_{2}=\operatorname{gcd}\left(n_{2}^{\prime}, n_{3}^{\prime}\right), \operatorname{gcd}\left(n_{2}^{\prime}, t_{1}\right)=\operatorname{gcd}\left(n_{3}^{\prime}, t_{2}\right)=1, \operatorname{gcd}\left(t_{3}, t_{4}\right)=1$, and $t_{3} \cdot n_{1}^{\prime}+t_{4} \cdot n_{2}^{\prime} \cdot n_{3}^{\prime}=n_{1}^{\prime} \cdot n_{2}^{\prime} \cdot n_{3}^{\prime}-1$.

Proof. If $t_{1}+t_{2}=\operatorname{gcd}\left(n_{2}^{\prime}, n_{3}^{\prime}\right)$ and $\operatorname{gcd}\left(n_{2}^{\prime}, t_{1}\right)=\operatorname{gcd}\left(n_{3}^{\prime}, t_{2}\right)=1$ for some positive integers $t_{1}, t_{2}$ then according to Theorem $1.1 C_{n_{2}^{\prime}} \square C_{n_{3}^{\prime}}$ has a Hamilton cycle. Hence, $C_{n_{1}^{\prime}} \square C_{n_{2}^{\prime}} \square C_{n_{3}^{\prime}}$ contains a spanning Cartesian product of directed cycles $H=$ $C_{n_{1}^{\prime}} \square C_{n_{2}^{\prime} \cdot n_{3}^{\prime}}$. By Lemma $2.1 H \backslash\{v\}$ has a Hamilton cycle if $\operatorname{gcd}\left(t_{3}, t_{4}\right)=1$ with $t_{3} \cdot n_{1}^{\prime}+t_{4} \cdot n_{2}^{\prime} \cdot n_{3}^{\prime}=n_{1}^{\prime} \cdot n_{2}^{\prime} \cdot n_{3}^{\prime}-1$. Furthermore, $C_{n_{1}^{\prime}} \square C_{n_{2}^{\prime}} \square C_{n_{3}^{\prime}} \cong C_{n_{1}} \square C_{n_{2}} \square C_{n_{3}}$ and according to Theorem $1.4 C_{n_{1}^{\prime}} \square C_{n_{2}^{\prime}} \square C_{n_{3}^{\prime}}$ contains a Hamilton cycle, which completes the proof.

By extension and similar approach, we can also give a sufficient condition for the Cartesian product of $k \geq 4$ directed cycles to be hyper-Hamiltonian as follows.

Theorem 3.2. Let $G=C_{n_{1}} \square C_{n_{2}} \square \cdots \square C_{n_{k}}$ be a Cartesian product of $k \geq 4$ directed cycles. Let $j$ be a positive integer such that $2 \neq j<\frac{k}{2}$. $G$ is hyper-Hamiltonian if there is a permutation $\left(n_{1}^{\prime}, n_{2}^{\prime}, \ldots, n_{k}^{\prime}\right)$ of $\left(n_{1}, n_{2}, \ldots, n_{k}\right)$ and there exist positive integers $t_{1}, t_{2}$ that satisfy $\operatorname{gcd}\left(t_{1}, t_{2}\right)=1$, and $t_{1} \cdot n_{1}^{\prime} \cdot n_{2}^{\prime} \cdots n_{j}^{\prime}+t_{2} \cdot n_{j+1}^{\prime} \cdot n_{j+2}^{\prime} \cdots n_{k}^{\prime}=n_{1}^{\prime} \cdot n_{2}^{\prime} \cdots n_{k}^{\prime}-1$.

Proof. Since $j \neq 2$ and $j<\frac{k}{2}$ then according to Theorem 1.4 $C_{n_{1}^{\prime}} \square C_{n_{2}^{\prime}} \square \cdots \square C_{n_{j}^{\prime}}$ and $C_{n_{j+1}^{\prime}} \square C_{n_{j+2}^{\prime}} \square \cdots \square C_{n_{k}^{\prime}}$ are Hamiltonian. Hence, $G^{\prime}=C_{n_{1}^{\prime}} \square C_{n_{2}^{\prime}} \square \cdots \square C_{n_{k}^{\prime}}$ contains a spanning Cartesian product of cycles $H=C_{n_{1}^{\prime} \cdot n_{2}^{\prime} \cdots n_{j}^{\prime}} \square C_{n_{j+1}^{\prime} \cdot n_{j+2}^{\prime} \cdots n_{k}^{\prime}}$. By Lemma $2.1 H \backslash\{v\}$ has a Hamilton cycle if $\operatorname{gcd}\left(t_{1}, t_{2}\right)=1$ with $t_{1} \cdot n_{1}^{\prime} \cdot n_{2}^{\prime} \cdots n_{j}^{\prime}+t_{2} \cdot n_{j+1}^{\prime} \cdot n_{j+2}^{\prime} \cdots n_{k}^{\prime}=n_{1}^{\prime} \cdot n_{2}^{\prime} \cdots n_{k}^{\prime}-1$. Furthermore, $G^{\prime} \cong G$ and according to Theorem $1.4 G^{\prime}$ contains a Hamilton cycle, which completes the proof.

While Theorems 3.1 and 3.2 give sufficient conditions for the Cartesian product of directed cycles $G$ to be hyperHamiltonian based on computing relatively prime integers, which would require executing Euclid's algorithm in nontrivial cases, the following special case of Theorem 3.2 can determine if $G$ is hyper-Hamiltonian more efficiently.

Corollary 3.1. Let $G=C_{n_{1}} \square C_{n_{2}} \square \cdots \square C_{n_{k}}$ be a Cartesian product of $k \geq 4$ directed cycles, where $n_{1}=2$ and $n_{2}, n_{3}, \ldots, n_{k}$ odd. Then $G$ is hyper-Hamiltonian.

Proof. By the same argument as in proof of Theorem 3.2, $G$ contains a spanning Cartesian product of two directed cycles $H=C_{2} \square C_{n_{2} \cdot n_{3} \cdots n_{k}}$. Furthermore, there is a positive integer $t$ such that $2 t-1=n_{2} \cdot n_{3} \cdots n_{k}$. By Theorem $1.2 H$ is hypo-Hamiltonian. So, $G \backslash\{v\}$ has a Hamilton cycle. Hence, according to Theorem $1.4 G$ is hyper-Hamiltonian. 
Finally, consider special cases when for $k \geq 4$ there exist positive integers $t_{1}$ and $t_{2}$ such that $\operatorname{gcd}\left(n_{1}^{\prime}, n_{2}^{\prime}\right)=t_{1}+t_{2}$ and $\operatorname{gcd}\left(n_{1}^{\prime}, t_{1}\right)=\operatorname{gcd}\left(n_{2}^{\prime}, t_{2}\right)=1$, where $\left(n_{1}^{\prime}, n_{2}^{\prime}, \ldots, n_{k}^{\prime}\right)$ is a permutation of $\left(n_{1}, n_{2}, \ldots, n_{k}\right)$. First, for $k=4$.

Theorem 3.3. $C_{n_{1}} \square C_{n_{2}} \square C_{n_{3}} \square C_{n_{4}}$ is hyper-Hamiltonian if there is a permutation $\left(n_{1}^{\prime}, n_{2}^{\prime}, n_{3}^{\prime}, n_{4}^{\prime}\right)$ of $\left(n_{1}, n_{2}, n_{3}, n_{4}\right)$ and there exist positive integers $t_{1}, t_{2}, t_{3}, t_{4}, t_{5}, t_{6}$ so that $t_{1}+t_{2}=\operatorname{gcd}\left(n_{1}^{\prime}, n_{2}^{\prime}\right), \operatorname{gcd}\left(n_{1}^{\prime}, t_{1}\right)=\operatorname{gcd}\left(n_{2}^{\prime}, t_{2}\right)=1, t_{3}+t_{4}=\operatorname{gcd}\left(n_{3}^{\prime}, n_{4}^{\prime}\right)$, $\operatorname{gcd}\left(n_{3}^{\prime}, t_{3}\right)=\operatorname{gcd}\left(n_{4}^{\prime}, t_{4}\right)=1, \operatorname{gcd}\left(t_{5}, t_{6}\right)=1$, and $t_{5} \cdot n_{1}^{\prime} \cdot n_{2}^{\prime}+t_{6} \cdot n_{3}^{\prime} \cdot n_{4}^{\prime}=n_{1}^{\prime} \cdot n_{2}^{\prime} \cdot n_{3}^{\prime} \cdot n_{4}^{\prime}-1$.

Proof. If $t_{1}+t_{2}=\operatorname{gcd}\left(n_{1}^{\prime}, n_{2}^{\prime}\right), \operatorname{gcd}\left(n_{1}^{\prime}, t_{1}\right)=\operatorname{gcd}\left(n_{2}^{\prime}, t_{2}\right)=1, t_{3}+t_{4}=\operatorname{gcd}\left(n_{3}^{\prime}, n_{4}^{\prime}\right), \operatorname{gcd}\left(n_{3}^{\prime}, t_{3}\right)=\operatorname{gcd}\left(n_{4}^{\prime}, t_{4}\right)=1$ for some positive integers $t_{1}, t_{2}, t_{3}, t_{4}$ then according to Theorem $1.1 C_{n_{1}^{\prime}} \square C_{n_{2}^{\prime}}$ and $C_{n_{3}^{\prime}} \square C_{n_{4}^{\prime}}$ have Hamilton cycles. Hence, $G=$ $C_{n_{1}^{\prime}} \square C_{n_{2}^{\prime}} \square C_{n_{3}^{\prime}} \square C_{n_{4}^{\prime}}$ contains a spanning Cartesian product of directed cycles $H=C_{n_{1}^{\prime} \cdot n_{2}^{\prime}} \square C_{n_{3}^{\prime} \cdot n_{4}^{\prime}}$. By Lemma $2.1 H \backslash\{v\}$ has a Hamilton cycle if $\operatorname{gcd}\left(t_{5}, t_{6}\right)=1$ with $t_{5} \cdot n_{1}^{\prime} \cdot n_{2}^{\prime}+t_{6} \cdot n_{3}^{\prime} \cdot n_{4}^{\prime}=n_{1}^{\prime} \cdot n_{2}^{\prime} \cdot n_{3}^{\prime} \cdot n_{4}^{\prime}-1$. Furthermore, $G \cong C_{n_{1}} \square C_{n_{2}} \square C_{n_{3}} \square C_{n_{4}}$ and according to Theorem $1.4 G$ contains a Hamilton cycle, which completes the proof.

Now, for $k>4$, one has the following theorem.

Theorem 3.4. Let $G=C_{n_{1}} \square C_{n_{2}} \square \cdots \square C_{n_{k}}$ be a Cartesian product of $k \geq 5$ directed cycles. $G$ is hyper-Hamiltonian if there is a permutation $\left(n_{1}^{\prime}, n_{2}^{\prime}, \cdots, n_{k}^{\prime}\right)$ of $\left(n_{1}, n_{2}, \cdots, n_{k}\right)$ and there exist positive integers $t_{1}, t_{2}, t_{3}, t_{4}$ so that $t_{1}+t_{2}=\operatorname{gcd}\left(n_{1}^{\prime}, n_{2}^{\prime}\right)$, $\operatorname{gcd}\left(n_{1}^{\prime}, t_{1}\right)=\operatorname{gcd}\left(n_{2}^{\prime}, t_{2}\right)=1, \operatorname{gcd}\left(t_{3}, t_{4}\right)=1$, and $t_{3} \cdot n_{1}^{\prime} \cdot n_{2}^{\prime}+t_{4} \cdot n_{3}^{\prime} \cdot n_{4}^{\prime} \cdots n_{k}^{\prime}=n_{1}^{\prime} \cdot n_{2}^{\prime} \cdots n_{k}^{\prime}-1$.

Proof. If $t_{1}+t_{2}=\operatorname{gcd}\left(n_{1}^{\prime}, n_{2}^{\prime}\right)$ and $\operatorname{gcd}\left(n_{1}^{\prime}, t_{1}\right)=\operatorname{gcd}\left(n_{2}^{\prime}, t_{2}\right)=1$ for some positive integers $t_{1}, t_{2}$ then according to Theorem $1.1 C_{n_{1}^{\prime}} \square C_{n_{2}^{\prime}}$ has a Hamilton cycle. Furthermore, since $k \geq 5$ then according to Theorem $1.4 C_{n_{3}^{\prime}} \square C_{n_{4}^{\prime}} \square \cdots \square C_{n_{k}^{\prime}}$ also has a Hamilton cycle. Hence, $G^{\prime}=C_{n_{1}^{\prime}} \square C_{n_{2}^{\prime}} \square \cdots \square C_{n_{k}^{\prime}}$ contains a spanning Cartesian product of directed cycles $H=$ $C_{n_{1}^{\prime} \cdot n_{2}^{\prime}} \square C_{n_{3}^{\prime} \cdot n_{4}^{\prime} \cdots n_{k}^{\prime}}$. By Lemma $2.1 H \backslash\{v\}$ has a Hamilton cycle if $\operatorname{gcd}\left(t_{3}, t_{4}\right)=1$ with $t_{3} \cdot n_{1}^{\prime} \cdot n_{2}^{\prime}+t_{4} \cdot n_{3}^{\prime} \cdot n_{4}^{\prime} \cdots n_{k}^{\prime}=n_{1}^{\prime} \cdot n_{2}^{\prime} \cdots n_{k}^{\prime}-1$. Furthermore, $G^{\prime} \cong C_{n_{1}} \square C_{n_{2}} \square \cdots \square C_{n_{k}}$ and according to Theorem $1.4 G$ contains a Hamilton cycle, which completes the proof.

\section{Acknowledgment}

I would like to thank the anonymous referees for valuable comments and suggestions, which resulted in improved presentation of this paper.

\section{References}

[1] Z. R. Bogdanowicz, On hyper-Hamiltonian Cartesian product of undirected cycles, Discrete Math. Lett. 7 (2021) 11-13.

[2] Z. R. Bogdanowicz, Hyper-Hamiltonian Circulants, Electron. J. Graph Theory Appl. 9 (2021) 185-193.

[3] S. J. Curran, D. Witte, Hamilton paths in Cartesian products of directed cycles, Ann. Discrete Math. 27 (1985) 35-74.

[4] R. R. Del-Vecchio, C. T. M. Vinagre, G. B. Pereira, Hyper-Hamiltonicity in graphs: some sufficient conditions, Electron. Notes Discrete Math. 62 (2017) $165-170$

[5] T. Mai, J. Wang, L. Hsu, Hyper-Hamiltonian generalized Petersen graphs, Comput. Math. Appl. 55 (2008) $2076-2085$.

[6] L. E. Penn, D. Witte, When the Cartesian product of two directed cycles is hypo-Hamiltonian, J. Graph Theory 7 (1983) $441-443$.

[7] R. A. Rankin, A campanological problem in group theory, Proc. Camb. Philos. Soc. 44 (1948) 17-25.

[8] C. Thomassen, Hypohamiltonian graphs and digraphs, In: Y. Alavi, D. R. Lick (Eds.) Theory and Applications of Graphs, Lecture Notes in Mathematics: Volume 642, Springer, Berlin, 1976, pp. 557-571.

[9] W. T. Trotter, P. Erdős, When the Cartesian product of directed cycles is Hamiltonian, J. Graph Theory 2 (1978) $137-142$. 